\title{
Cerebrospinal fluid neurogranin concentration in neurodegeneration: relation to clinical phenotypes and neuropathology
}

\author{
Erik Portelius ${ }^{1,2}$ - Bob Olsson ${ }^{1,2} \cdot$ Kina Höglund $^{1,2} \cdot$ Nicholas C. Cullen $^{1} \cdot$ Hlin Kvartsberg ${ }^{1} \cdot$ Ulf Andreasson $^{1,2}$. \\ Henrik Zetterberg ${ }^{1,2,3,4}$. Åsa Sandelius ${ }^{1}$. Leslie M. Shaw ${ }^{5}$ - Virginia M. Y. Lee ${ }^{5}$. David J. Irwin ${ }^{6}$. Murray Grossman ${ }^{6}$. \\ Daniel Weintraub ${ }^{7,8} \cdot$ Alice Chen-Plotkin $^{6}$. David A. Wolk ${ }^{6} \cdot$ Leo McCluskey $^{6} \cdot$ Lauren Elman $^{6}$. Jennifer McBride ${ }^{5}$. \\ Jon B. Toledo ${ }^{5,9} \cdot$ John Q. Trojanowski ${ }^{5} \cdot$ Kaj Blennow $^{1,2}$
}

Received: 13 December 2017 / Revised: 19 April 2018 / Accepted: 21 April 2018 / Published online: 26 April 2018

(c) The Author(s) 2018

\begin{abstract}
Neurogranin $(\mathrm{Ng})$ is a post-synaptic protein that previously has been shown to be a biomarker for synaptic function when measured in cerebrospinal fluid (CSF). The CSF concentration of $\mathrm{Ng}$ is increased in Alzheimer's disease dementia (ADD), and even in the pre-dementia stage. In this prospective study, we used an enzyme-linked immunosorbent assay that quantifies $\mathrm{Ng}$ in CSF to test the performance of $\mathrm{Ng}$ as a marker of synaptic function. In 915 patients, CSF Ng was evaluated across several different neurodegenerative diseases. Of these 915 patients, 116 had a neuropathologically confirmed definitive diagnosis and the relation between CSF Ng and topographical distribution of different pathologies in the brain was evaluated. CSF Ng was specifically increased in ADD compared to eight other neurodegenerative diseases, including Parkinson's disease $(p<0.0001)$, frontotemporal dementia $(p<0.0001)$, and amyotrophic lateral sclerosis $(p=0.0002)$. Similar results were obtained in neuropathologically confirmed cases. Using a biomarker index to evaluate whether CSF Ng contributed diagnostic information to the core AD CSF biomarkers (amyloid $\beta(\mathrm{A} \beta)$, t-tau, and p-tau), we show that $\mathrm{Ng}$ significantly increased the discrimination between AD and several other disorders. Higher CSF Ng levels were positively associated with greater $\mathrm{A} \beta$ neuritic plaque (Consortium to Establish a Registry for Alzheimer's Disease (CERAD) neuritic plaque score, $p=0.0002$ ) and tau tangle pathology (Braak neurofibrillary tangles staging, $p=0.0007$ ) scores. In the hippocampus and amygdala, two brain regions heavily affected in ADD with high expression of $\mathrm{Ng}$, CSF Ng was associated with plaque ( $p=0.0006$ and $p<0.0001$ ), but not with tangle, $\alpha$-synuclein, or TAR DNA-binding protein 43 loads. These data support that CSF Ng is increased specifically in ADD, that high CSF Ng concentrations likely reflect synaptic dysfunction and that CSF Ng is associated with $\beta$-amyloid plaque pathology.
\end{abstract}

Keywords Alzheimer's disease $\cdot$ Neurogranin $\cdot$ Biomarker $\cdot$ Neuropathology $\cdot$ Cerebrospinal fluid

\section{Introduction}

Synaptic density, and thus synaptic protein expression, is highest in the associative cortical areas, probably reflecting cognitive processing [4]. In Alzheimer's disease dementia

Erik Portelius and Bob Olsson contributed equally to this work.

Electronic supplementary material The online version of this article (https://doi.org/10.1007/s00401-018-1851-x) contains supplementary material, which is available to authorized users.

Erik Portelius

erik.portelius@neuro.gu.se

Extended author information available on the last page of the article
(ADD) these brain regions show synaptic dysfunction, degeneration, and loss; synaptic pathology occurs early in the disease process, perhaps even earlier than neuronal degeneration and loss $[2,10]$, which is supported by studies of a tauopathy mouse model [56]. In addition, neuropathological studies have shown that this synaptic dysfunction is more linked to memory dysfunction than plaque and tangle pathologies, which are the two major pathological hallmarks of $\operatorname{ADD}[3,9,29,48]$.

Neurogranin $(\mathrm{Ng})$ is a neuronal protein that is highly expressed in the cortex, hippocampus, and amygdala, with the highest concentrations at the dendritic spines [16, 42]. Since the discovery that $\mathrm{Ng}$ is present in CSF [8], and that levels are increased in ADD [50], several recent studies have 
reported higher cerebrospinal fluid (CSF) $\mathrm{Ng}$ concentration in ADD and mild cognitive impairment (MCI) patients compared to cognitively unimpaired elderly subjects [26-28, 43]. Further, other studies suggest that increased CSF Ng concentrations may be specific for ADD [25, 41, 53].

Recently, we showed that CSF Ng concentrations can predict the rate of cognitive decline in prodromal ADD and conversion from MCI to ADD [39]. Accordingly, CSF Ng seems to be a novel biomarker reflecting ADD-associated synaptic dysfunction, which may be used to improve early diagnosis and prognostication, as well as monitoring effects of disease-modifying drug candidates on synaptic status.

In CSF, $\mathrm{Ng}$ is present as several endogenous peptides of different lengths, as well as full-length protein (78 amino acids) [27]. To further explore CSF $\mathrm{Ng}$ as a biomarker for ADD, we optimized and validated an enzyme-linked immunosorbent assay (ELISA) that quantifies C-terminal $\mathrm{Ng}$ peptides and full-length $\mathrm{Ng}$ protein in CSF. Here we present results on CSF $\mathrm{Ng}$ in a very large clinical cohort including several different neurodegenerative diseases with a subset followed to autopsy for determination of the neuropathology definitive diagnoses. The specific hypotheses tested were that increased CSF Ng is specific to ADD, and that patients with autopsy-confirmed ADD have higher CSF Ng concentrations compared to patients with dementia with Lewy bodies (DLB), frontotemporal dementia (FTD), progressive supranuclear palsy (PSP) or amyotrophic lateral sclerosis (ALS). Last, we wanted to explore the relationship between CSF Ng concentrations and the topographical distribution of neuritic plaques and tau tangles in the brain.

\section{Materials and methods}

\section{Subjects}

Subjects included 75 controls (CTRL), 114 MCI, 397 ADD, 6 posterior cortical atrophy (PCA), 96 FTD [46 behavioral variant FTD (bvFTD), 12 logopenic variant primary progressive aphasia (lvPPA), 20 non-fluent variant primary progressive aphasia (nfvPPA), 18 semantic variant PPA (svPPA)], 68 ALS, 37 Parkinson's disease with normal cognition (PD), 19 PD with MCI (PD MCI), 29 PD with dementia (PDD), 33 DLB, 21 corticobasal syndrome (CBS), and 20 PSP patients. Recruitment of the patients and diagnostic criteria for the groups have been described previously in detail $[20,52,55]$. Demographic and biomarker characteristics of the patients included in the study are shown in Table 1. Patients were clinically evaluated at each clinical core [52] and current clinical criteria were used for diagnosis of AD [31, 32], bvFTD [40], PPA [15], CBS [1], PSP [17], ALS [46] and DLB [30]. The onset of disease was defined by the year reported by patients/family of functional impairment in cognitive/motor features. Standardized neuropsychological assessments were collected by trained examiners at each center. All patients were evaluated at the clinical cores at the University Of Pennsylvania Perelman School Of Medicine including the Penn Alzheimer's disease core center, Frontotemporal degeneration center, Udall Center for Parkinson's' disease research and Amyotrophic lateral sclerosis center. CSF samples were collected in a standardized manner using the standard operating procedures of ADNI as described (http://www.adni-info.org/). Out of the 915 subjects included in the study, 116 had a definitive diagnosis by neuropathology. The definitive diagnostic groups included $\operatorname{ADD}(n=75), \operatorname{DLB}(n=16), \operatorname{FTD}(n=12), \operatorname{ALS}(n=7)$, and PSP $(n=6)$ subjects. The neuropathological data and criteria have been described elsewhere [19, 37, 51, 52]. See Online Resource 1 for demographics.

Genomic DNA was extracted from peripheral blood before death or frozen brain samples postmortem as described elsewhere [23]. APOE allele status was defined using two SNPs (rs7412 and rs429358) which were genotyped by TaqMan allelic discrimination assays (ThermoFisher, USA).

Braak tau neurofibrillary tangle staging (PHF-1) and the Consortium to Establish a Registry for Alzheimer's Disease (CERAD) neuritic plaque score (thioflavin stain) were used to classify ADD neuropathology into four groups as described previously [19, 21]: no (or negligible) ADD neuropathology (0), low-level ADD (1), intermediate-level ADD (2), and high-level ADD (3). For the determination of neuronal loss, the brain sections were stained with hematoxylin-eosin and Thal staging (nab228) $(n=114)$ was performed as described elsewhere [52]. An ABC score that incorporates histopathologic assessments of $A \beta$ deposits (A), staging of neurofibrillary tangles (B), and scoring of neuritic plaques (C) was calculated as described [37].

Postmortem examination and scoring of tau, A $\beta$, $\alpha$-synuclein, and TAR DNA-binding protein 43 (TDP-43) pathology were performed on amygdala and cornu ammonis/ subiculum-hippocampus as described elsewhere [52].

The Alzheimer's Disease Core Center (ADCC), Penn Memory Center, the Frontotemporal Degeneration Center, the ALS Center, the Parkinson's Disease and Movement Disorder Clinic, and the Penn Udall Center for Parkinson's Research each have protocols approved by the institutional review board to recruit patients, along with their clinical data, into research studies. In addition, these centers invite patients to participate in the brain donation program.

\section{CSF measurements}

The generation and purification of the anti-Ng monoclonal antibodies (Mab) NG22 (epitope 63-75) and NG2 (epitope 52-63) were performed as described previously [44]. 


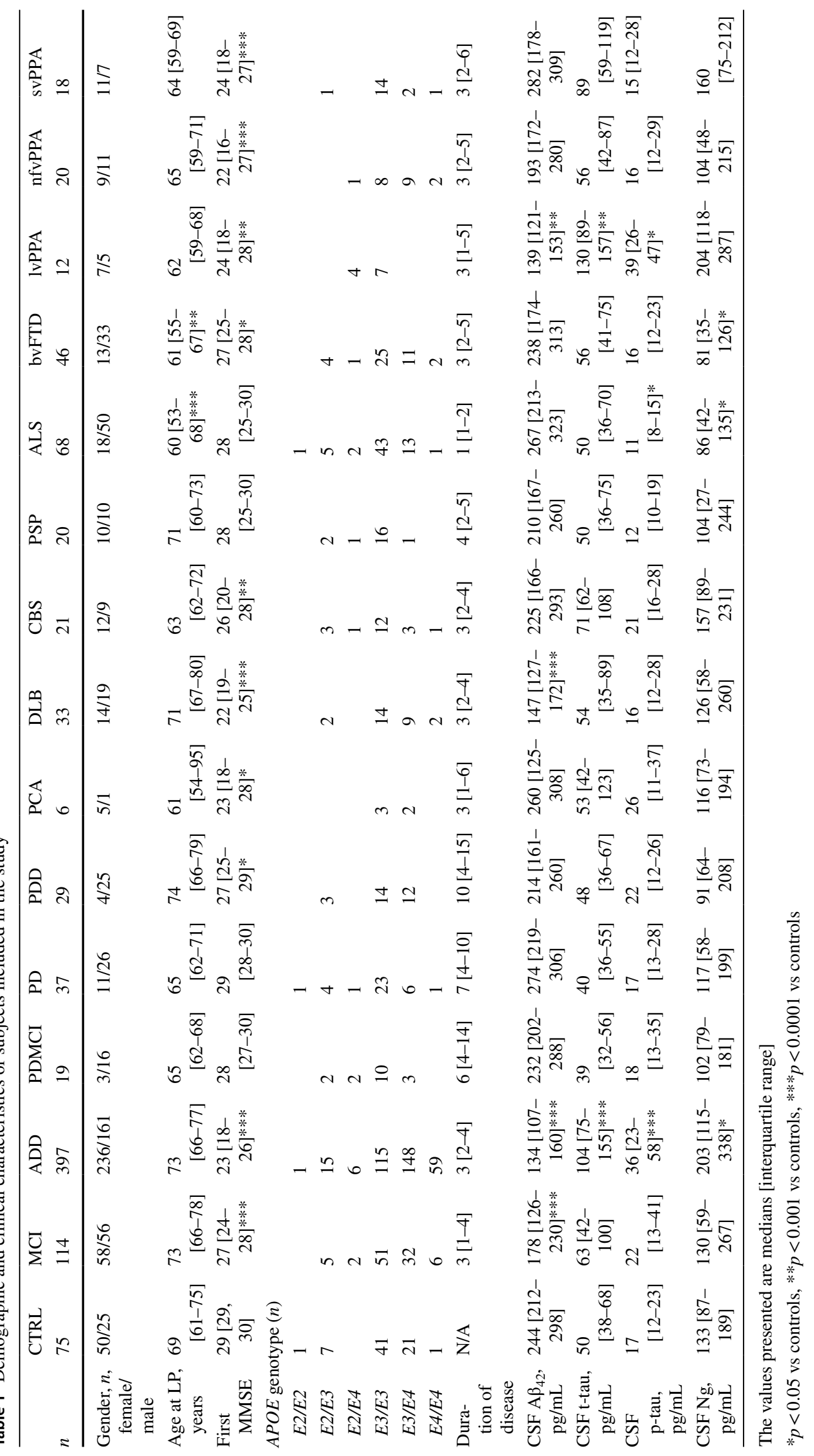


96-well plates were coated with $3 \mu \mathrm{g} / \mathrm{mL}$ (100 $\mu \mathrm{L} /$ well) of the Mab NG22 in $50 \mathrm{mM}$ bicarbonate buffer ( $\mathrm{pH} 9.6$ ) and incubated overnight $\left(16-18 \mathrm{~h}\right.$ ) at $+4{ }^{\circ} \mathrm{C}$. After washing four times with $0.05 \%$ Tween 20 in PBS (PBS-Tween) $(350 \mu \mathrm{L} /$ well), the remaining protein binding sites were blocked with $1 \%$ bovine serum albumin (BSA) in PBS $(0.01 \mathrm{M}$ phosphate buffer, $0.14 \mathrm{M} \mathrm{NaCl}, \mathrm{pH} 7.4$ ) for $1 \mathrm{~h}$ at $+20^{\circ} \mathrm{C}$ $\left(250 \mu \mathrm{L} /\right.$ well). Coated plates were then stored at $-20{ }^{\circ} \mathrm{C}$. Prior to ELISA measurement, the plates were thawed and then washed with PBS- $-0.05 \%$ Tween four times $(350 \mu \mathrm{L}$ per well and wash), followed by addition of $100 \mu \mathrm{L}$ of samples, controls, and calibrators to the plate. Recombinant fulllength $\mathrm{Ng}$ protein with a GST-tag was used as calibrator. The calibration curve ranged from 25.9 to $3310 \mathrm{pg} / \mathrm{mL}$ (1:2 dilutions in $0.5 \%$ octyl beta-D-glucopyranoside, $1 \%$ BSA in PBS). The samples, blanks, and calibrators were incubated overnight at $+4{ }^{\circ} \mathrm{C}$. Next day the plates were washed and then incubated for $1 \mathrm{~h}$ at $350 \mathrm{rpm}$ (room temperature) with the detector antibody, biotinylated NG2 $(2.7 \mu \mathrm{g} / \mathrm{mL})$ in $1 \%$ BSA in PBS-Tween (100 $\mu \mathrm{L} /$ well). After another washing step, the plates were incubated for $30 \mathrm{~min}$ (room temperature) with $100 \mu \mathrm{L} /$ well of enhanced streptavidin-HRP (Kem En Tech \#4740 N) diluted 1:20 000 in 1\% BSA in PBS-Tween. The plates were then washed and color reaction was started using $100 \mu \mathrm{L} /$ well of substrate (TMB one, ready to use, Kem En Tech \#4380A). After 20 min in dark the reaction was stopped using $100 \mu \mathrm{L} /$ well of $0.2 \mathrm{M}$ of $\mathrm{H}_{2} \mathrm{SO}_{4}$ and the absorbance was measured at $450 \mathrm{~nm}$ (reference wavelength $650 \mathrm{~nm})$ using an ELISA plate reader $\left(V_{\max }\right.$, Molecular Devices, USA). A fitted four-parameter logistic model was used as the calibration curve (SoftMax Pro v. 4.0, Molecular Devices, USA). The analyses were performed by board-certified laboratory technicians blinded to clinical information.

CSF collection, processing, and storage procedures have been described previously [45]. CSF A $\beta 42$, total-tau (t-tau), and phosphorylated tau (p-tau) were measured using the multiplex xMAP Luminex platform (Luminex Corp, Austin, TX, USA) with the INNOBIA AlzBio3 kit (Innogenetics, Ghent, Belgium) as described previously [45]. Subjects were classified as ADD biomarker positive or negative using previously established cutoffs (CSF A $\beta_{42}<192 \mathrm{pg} / \mathrm{mL}, \mathrm{CSF}$ $\mathrm{t}$-tau $>93 \mathrm{pg} / \mathrm{mL}$ ) that maximized the separation of autopsyconfirmed ADD cases with A $\beta$ pathology from controls without $A \beta$ pathology as described by Shaw et al. [45].

\section{Statistical analysis}

Statistical analyses were performed using GraphPad Prism 7 and the R programming language (version 3.4.3), while the biomarker index model was developed using Python 3.6. Because biomarker values were skewed, non-parametric tests were used. Differences between groups were assessed using the non-parametric Kruskal-Wallis test followed by Dunn's multiple-comparison test if significant. Because post hoc analysis involved a large number of comparisons, reported $p$ values were adjusted using Holm-Bonferroni procedure to control the family-wise error rate. The associations of $\mathrm{Ng}$ with the other CSF biomarkers A $\beta 42$, t-tau and p-tau were investigated with Spearman's rank correlation $\left(\mathrm{rho}_{\mathrm{s}}\right)$. All tests were two sided and significance threshold was set at $p<0.05$. We investigated the relationship between CSF Ng and MMSE change per year while adjusting for age, sex, and disease duration using partial Spearman's rank correlation. We tested this relationship in the whole study population, only the MCI group, and only the ADD group. Additionally, a biomarker index model was developed with the goal of accurately discriminating between two disease groups on the basis of their CSF biomarker measurements. The baseline model included CSF A $\beta 42$, CSF t-tau and CSF p-tau, and the $\mathrm{Ng}$ model included CSF Ng in addition to the three aforementioned CSF measurements. The discriminator itself is a support vector machine (SVM), see Online Resource 2 for a detailed description.

\section{Results}

\section{CSF Ng in AD and other diagnoses}

CSF Ng concentrations were significantly higher in ADD compared to both MCI $(p<0.0001)$ and CTRL $(p=0.0001)$ while the concentrations were similar in CTRL and MCI (Fig. 1a). Based on previously defined cutoff concentrations for t-tau (93 pg/mL) and A $\beta 1-42(192 \mathrm{pg} / \mathrm{mL})$ [45], subjects were classified as AD biomarker positive or negative by calculating a ratio between the two biomarkers (t-tau/A $\beta 1-42$ ). A value of $<0.48$ was considered as $\mathrm{AD}$ biomarker negative. When applying this cutoff on the CTRL group, the AD biomarker-positive CTRL subjects $(n=9)$ had significantly higher CSF Ng concentrations than the AD biomarkernegative CTRL group subjects ( $n=64, p=0.03$ ) (Fig. 1b). Subjects in the former group $(n=9)$ were excluded from the control group, as they were considered having preclinical AD pathology. Similarly, the AD biomarker-positive ADD and MCI groups both had significantly increased CSF $\mathrm{Ng}$ concentrations compared to the biomarker-negative group ( $p<0.0001$ for both) (Fig. 1c, d). In addition, AD biomarker-positive DLB patients had significantly increased $\mathrm{Ng}$ concentrations compared to biomarker-negative DLB and ADD subjects ( $p=0.0005$ and $p=0.002$, respectively) (Fig. 1e). The ADD and MCI biomarker-negative patients were excluded from further analysis $(n=83$ and $n=74$ for ADD and MCI, respectively). 

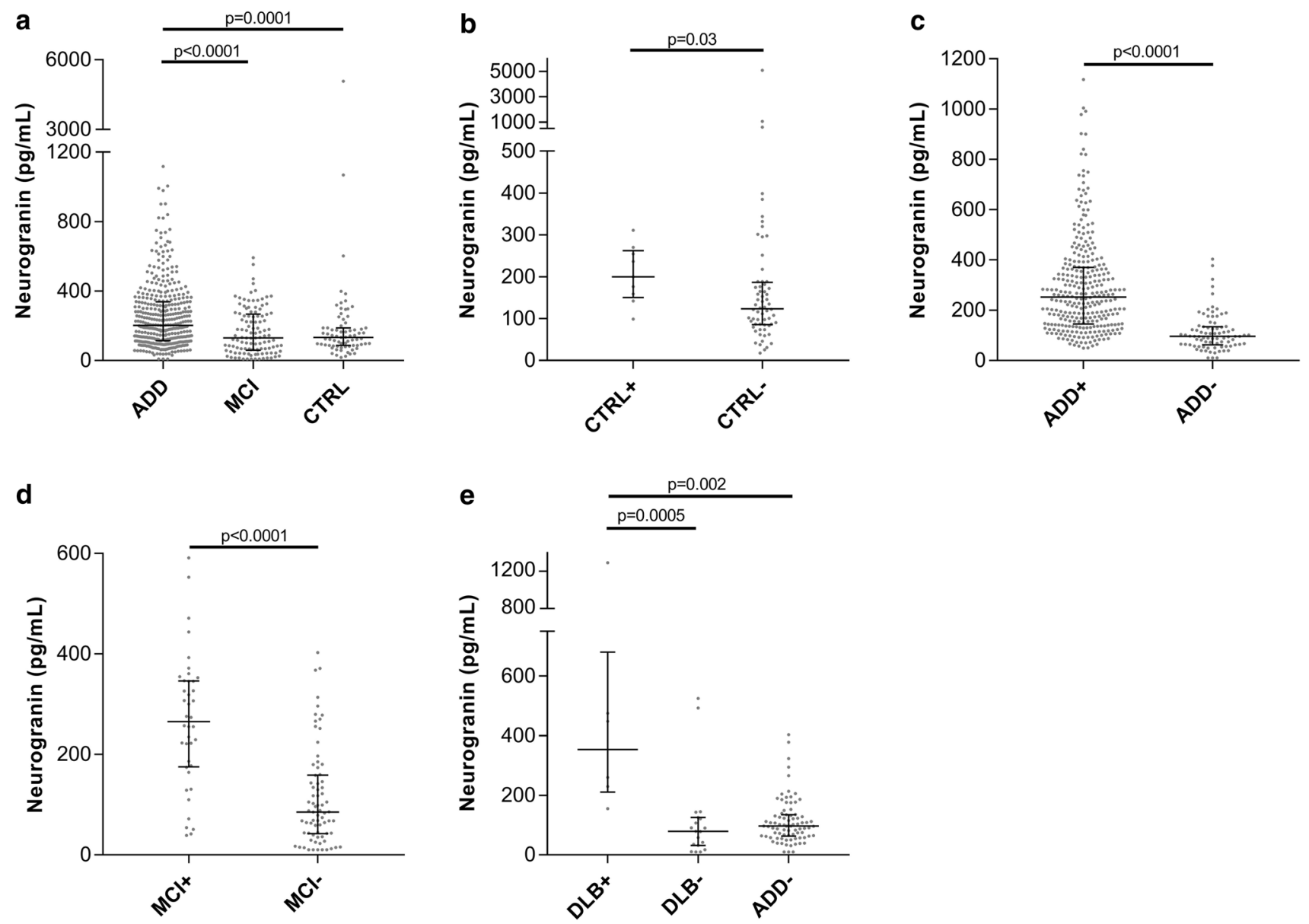

Fig. 1 a Scatterplots displaying the CSF $\mathrm{Ng}$ concentrations in ADD, MCI and CTRL. The CTRL (b), ADD (c) MCI (d) and DLB (e) groups were divided into biomarker positive $(+)$ or negative $(-)$ for ADD based on previously established cutoff concentrations for t-tau

Relative to ADD, the $\mathrm{Ng}$ concentrations were significantly lower in the PD $(p<0.0001)$, PD MCI $(p=0.005)$, $\operatorname{PDD}(p<0.0001)$, DLB $(p=0.002)$, CBS $(p=0.03)$, and PSP ( $p=0.004)$ (Fig. 2a). There were no significant differences in CSF Ng concentrations between the atypical parkinsonian diagnosis (CBS and PSP), DLB, and PD. The ADD group had significantly higher $\mathrm{Ng}$ concentrations compared to both FTD $(p<0.0001)$ and ALS $(p<0.0001)$ (Fig. 2b). FTD is a pathologically heterogeneous entity that includes several related disorders in which progressive degeneration of the frontal and temporal lobes is common $[15,40]$. On the basis of clinical phenotypes, we divided the FTD group into the following subgroups; bvFTD and PPA of which the latter can be further divided into nfvPPA, lvPPA, and svPPA [15]. Compared to ADD, nfvPPA, svPPA, and bvFTD had significantly lower CSF $\mathrm{Ng}$ concentrations $(p=0.0004, p=0.01$, and $p<0.0001)$ (Fig. 2c). Interestingly, lvPPA had significantly increased concentrations compared to bvFTD $(p<0.02)$ and similar and $A \beta 42$ [45]. The bars presented in the figures are medians with interquartile ranges and comparisons between groups were performed using Kruskal-Wallis test, followed by the Mann-Whitney $U$ test

CSF Ng concentrations as ADD (Fig. 2c). In contrast, bvFTD had a tendency towards decreased CSF Ng concentrations compared to CTRL but this did not reach statistical significance (Fig. 2c).

To test if CSF Ng contributed additional information in the context of discriminating between neurodegenerative disorders we developed a biomarker index model with the goal of accurately discriminating between two disease groups on the basis of their CSF biomarker measurements (A $\beta$, t-tau, $\mathrm{p}$-tau and $\mathrm{Ng}$ ). We found that $\mathrm{Ng}$ significantly increased the SVM model's ability to discriminate between numerous pairs of disorders. More specifically, the inclusion of $\mathrm{Ng}$ led to an increase in $24 \%$ accuracy in distinguishing between CTRL and bvFTD, $3.6 \%$ increase for MCI vs bvFTD, and $9.5 \%$ increase for PD vs bvFTD. Including $\mathrm{Ng}$ also led to a $7.7 \%$ increase in accuracy in distinguishing between MCI and PD, 6.4\% increase for MCI vs PD MCI, and $4.3 \%$ increase for MCI vs PDD (see Online Resource 3). 
Fig. 2 Scatterplots displaying the CSF Ng concentrations in a biomarker-positive ADD, PD, PD MCI, PDD, DLB CBD and PSP and $\mathbf{b}$ biomarker-positive ADD, FTD and ALS. c Scatterplots showing the $\mathrm{CSF} \mathrm{Ng}$ concentrations after that the FTD group was divided into the subgroups PPA log, PPA PNFA, PPA SD and bvFTD. The bars presented in the figures are medians with interquartile ranges and comparisons between groups were performed using Kruskal-Wallis test, followed by the Mann-Whitney $U$ test. The dashed lines represent the median for biomarker-negative CTRL

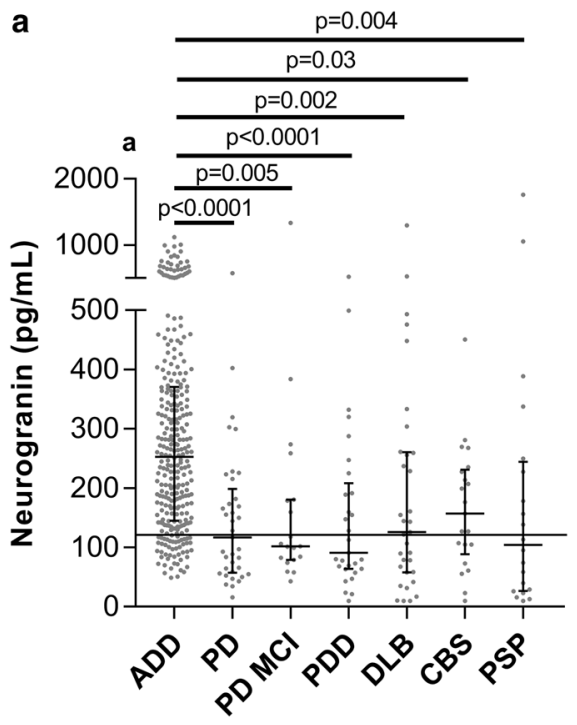

b

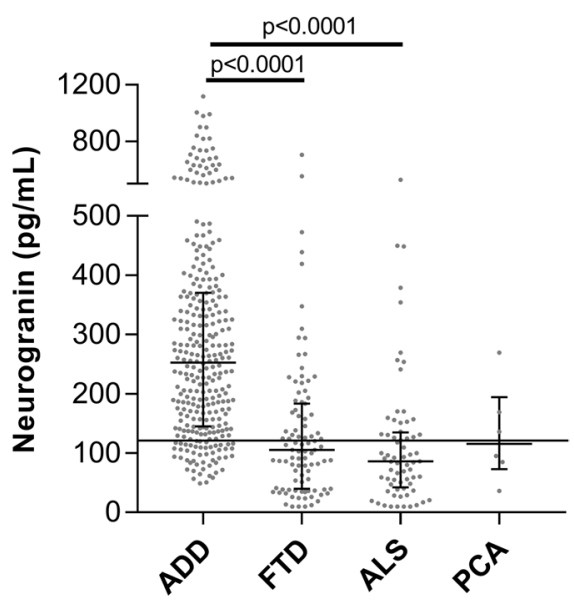

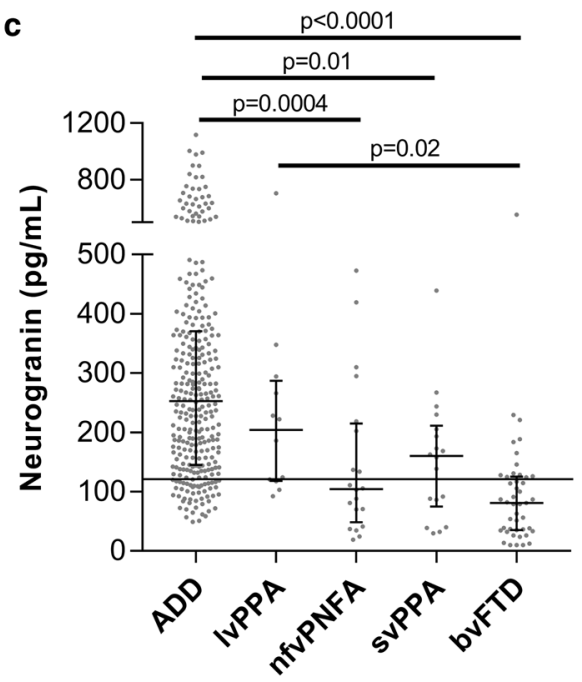

\section{CSF $\mathrm{Ng}$ in relation to tau and $\mathrm{A} \beta$}

t-tau correlated positively with CSF Ng in all diagnostic groups except in PSP, PCA and lvPPA (see Table 2 for rho $_{\mathrm{s}}$ and $p$ values). p-tau correlated with CSF Ng in CTRL $\left(\mathrm{rho}_{\mathrm{s}}=0.39\right)$, MCI $\left(\mathrm{rho}_{\mathrm{s}}=0.60\right)$, and ADD $\left(\mathrm{rho}_{\mathrm{s}}=0.56\right)(p<0.001$ for all groups $)$ as well in PD MCI $\left(\mathrm{rho}_{\mathrm{s}}=0.52, p<0.05\right)$, DLB $\left(\mathrm{rho}_{\mathrm{s}}=0.56, p<0.01\right)$, and bvFTD $\left(\right.$ rho $\left._{\mathrm{s}}=0.49, p<0.01\right)$ but not in the other diagnoses (Table 2). CSF Ng concentration showed a weak negative correlation with CSF A $\beta 42$ in ADD $\left(\mathrm{rho}_{\mathrm{s}}=-0.15\right.$, $p=0.004$ ) while a stronger positive correlation with CSF A $\beta 42$ was found in ALS $\left(\mathrm{rho}_{\mathrm{s}}=0.48, p<0.0001\right.$ ) (Fig. 3a, b). There were no correlations between CSF Ng and A $\beta 42$ in the other groups.

\section{CSF $\mathrm{Ng}$ in relation to MMSE}

While controlling for age, gender, and disease duration, we found a significant association between CSF Ng concentration and longitudinal decline in MMSE scores per year in the whole study population $\left(\mathrm{rho}_{\mathrm{s}}=0.17, p=0.001\right)$ (Fig. 4a). In the ADD group there was a suggestive relationship without covariate adjustment $\left(\mathrm{rho}_{\mathrm{s}}=0.12\right.$, $p=0.085$ ) which was no longer observed after covariate adjustment $\left(\mathrm{rho}_{\mathrm{s}}=0.07, p=0.3\right)$ (Fig. 4b). There was a significant relationship in the MCI group $\left(\mathrm{rho}_{\mathrm{s}}=0.45\right.$, $p=0.001$ ) (Fig. 4c). CSF Ng concentrations did not correlate with rates of change in MMSE in the FTD, ALS, or PD groups. 
Table 2 Correlations between CSF Ng and tau

\begin{tabular}{llc}
\hline Clinical diagnosis & $\mathrm{t}$-tau & $\mathrm{p}$-tau \\
\hline CTRL & $0.61 * * *$ & $0.39 * * *$ \\
MCI & $0.79 * * *$ & $0.60 * * *$ \\
ADD & $0.77^{* * *}$ & $0.56 * * *$ \\
PD MCI & $0.51^{*}$ & $0.52^{*}$ \\
PD & $0.75^{* * *}$ & 0.14 \\
PDD & $0.48^{* *}$ & 0.18 \\
PCA & 0.3 & -0.3 \\
DLB & $0.77 * * *$ & $0.56 * *$ \\
CBS & $0.87 * * *$ & 0.2 \\
PSP & 0.37 & -0.26 \\
ALS & $0.48^{* * *}$ & -0.097 \\
bvFTD & $0.57 * * *$ & $0.49 * *$ \\
lvPPA & 0.64 & 0.33 \\
nfvPPA & $0.76 * *$ & 0.19 \\
svPPA & $0.80^{* * *}$ & 0.35 \\
\hline
\end{tabular}

The values are Spearman's rank correlation coefficient

${ }^{*} p<0.05,{ }^{* *} p<0.01, * * * p<0.001$

\section{CSF $\mathrm{Ng}$ in relation to $\mathrm{APOE}$ genotype}

When grouping according to the number of $A P O E \varepsilon 4$ alleles, CSF Ng increased in a gene dose-dependent manner with the highest $\mathrm{Ng}$ concentrations in the group homozygous for the $\varepsilon 4$ allele (Fig. 5a). CSF Ng concentrations were also strongly associated with the number of copies of the $\varepsilon 4$ allele within the ADD group with the highest concentrations in the group having two copies of the $\varepsilon 4$ allele ( $p=0.002)$ (Fig. 5b). There was a trend towards increased CSF Ng concentrations with increasing number of $A P O E \& 4$ alleles within the MCI group, but this did not reach statistical significance (Fig. 5c).

\section{CSF $\mathrm{Ng}$ in relation to autopsy-confirmed patients}

In the autopsy-confirmed cases with definitive diagnoses, CSF Ng was significantly increased in ADD compared to DLB $(p=0.03)$, frontotemporal lobar degeneration FTLD $(p=0.006)$, and ALS $(p=0.03)$ (Fig. 6a). We also examined the association between CSF $\mathrm{Ng}$ and the burden of neurofibrillary tangles and plaques in the brain. Braak neurofibrillary staging and CERAD were used to classify the neuropathology into four groups as described previously [37] ranging from no AD to high-level AD pathology. In this analysis, CSF Ng was significantly increased in the high-level pathology AD group having most widespread tau $(p=0.0007)$ and $\mathrm{A} \beta$ plaque $(p=0.0002)$ pathology compared to no (or negligible) AD pathology (Fig. 6b, c).

Since Ng is highly expressed in the cerebral cortex, hippocampus and amygdala, which are the same brain regions that are affected in ADD [42], we investigated the relationship between CSF Ng concentrations and neuropathology findings in amygdala and hippocampus. While there were no associations between CSF $\mathrm{Ng}$ and tau neurofibrillary tangles, $\alpha$-synuclein, or TDP-43 load in amygdala or hippocampus, we found that higher $\mathrm{A} \beta$ plaque load in the amygdala and hippocampus correlated with increasing CSF $\mathrm{Ng}$ concentrations $(p<0.0001$ in amygdala and $p=0.0006$ in hippocampus) (Fig. 6d, e). In addition, we also found an association between CSF $\mathrm{Ng}$ concentrations and neuronal loss in the hippocampus ( $p=0.04$ ) (Fig. 6f) while there were no association between CSF $\mathrm{Ng}$ and neuronal loss in the amygdala.

Next we averaged CSF Ng values for subjects with postmortem evaluation of AD pathology according to a pathology classification table as previously described [37]. We then aggregated CSF Ng values over each pathology stage for each of the three classification schemes to understand the relationship between CSF Ng and each classification scheme individually. We found a significant difference in CSF Ng concentrations between the different pathology stages in the
Fig. 3 Correlations between CSF Ng concentrations and A 342 in clinically diagnosed ADD (a) and clinically diagnosed ALS (b) patients. The dashed lines represent the $95 \%$ confidence bands of the best-fit line. The associations were investigated with Spearman's rank correlation
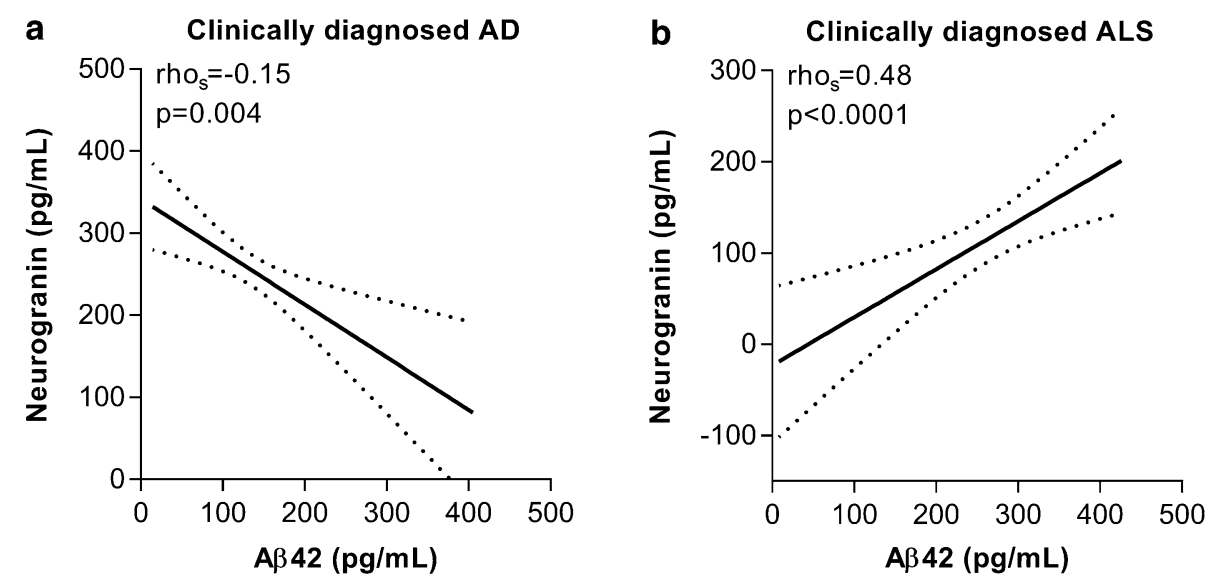
Fig. 4 Correlations between CSF Ng concentrations and loss in MMSE points/year in a all subjects included in the study, b clinically diagnosed ADD and $\mathbf{c}$ clinically diagnosed MCI. The dashed lines represent the $95 \%$ confidence bands of the best-fit line. The associations were investigated with Spearman's rank correlation
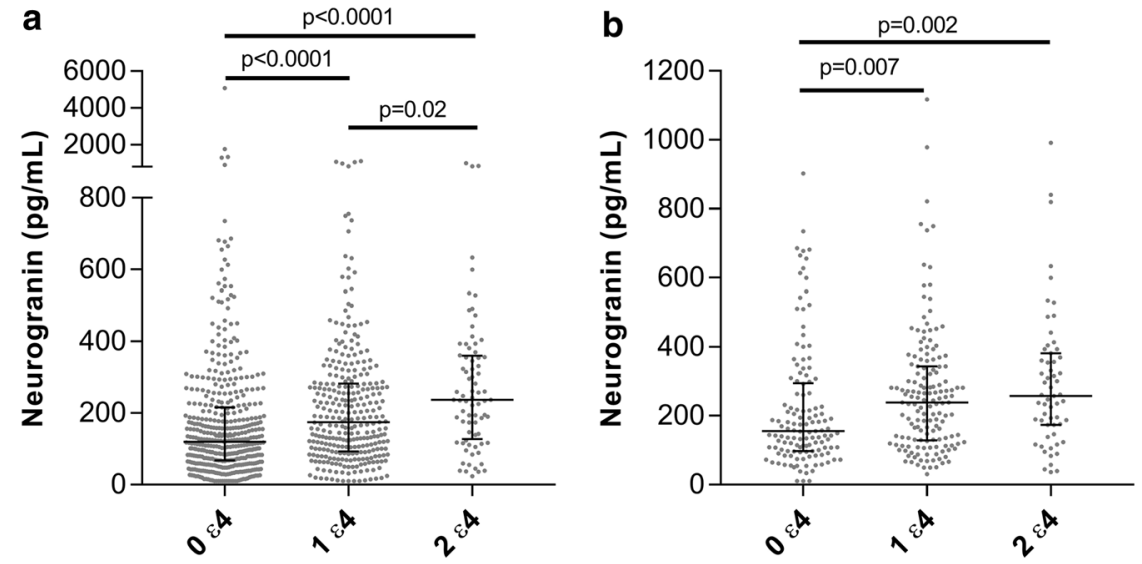

a All subjects included in the study

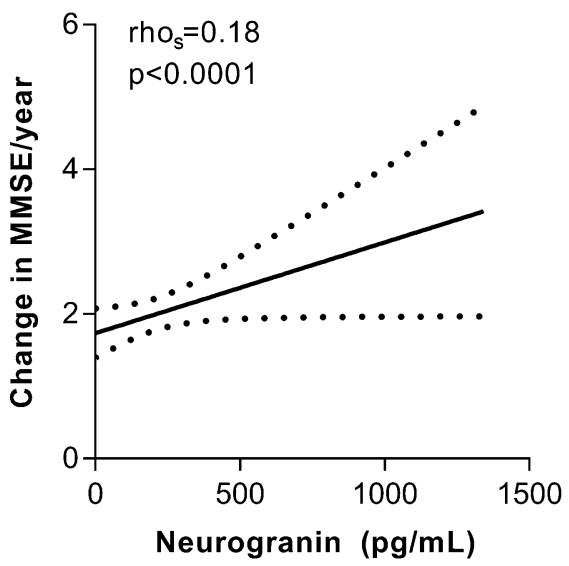

c Clinically diagnosed $\mathrm{MCl}$

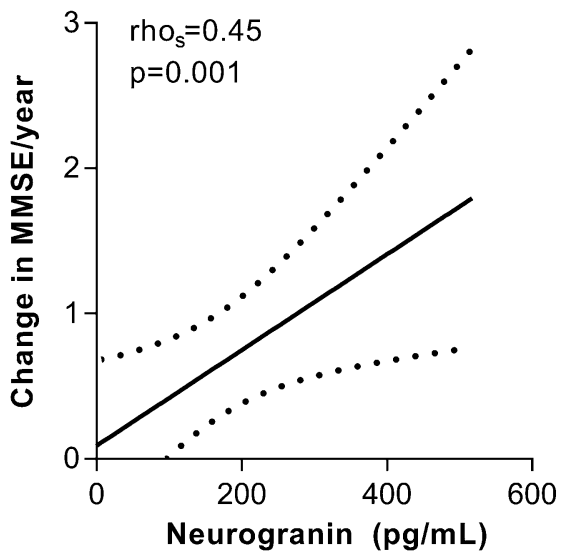

C

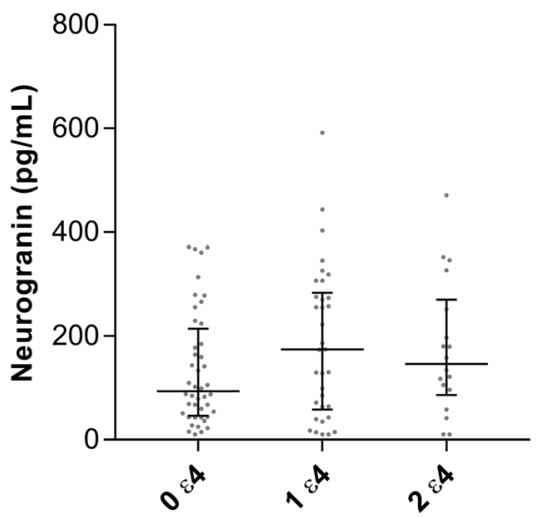

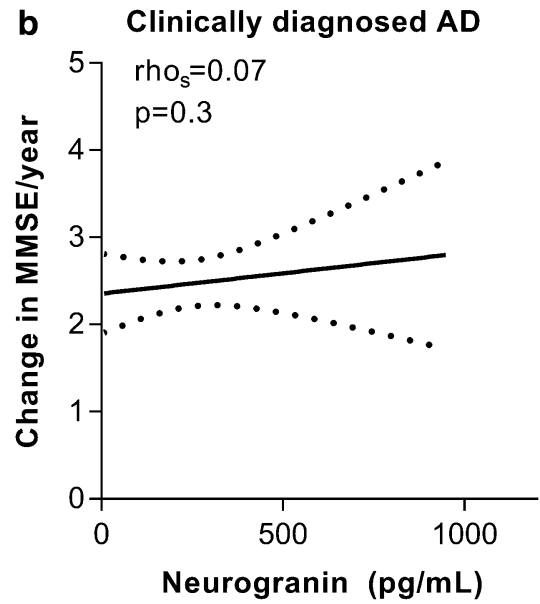

Fig. 5 Scatterplots displaying the CSF $\mathrm{Ng}$ concentrations in patients having zero, one or two APOE $\varepsilon 4$ alleles in a all subjects included in the study $\mathbf{b}$ clinically diagnosed ADD and $\mathbf{c}$ clinically diagnosed

Thal stage $(H=22.75, p<0.0001)$, as well as in the Braak stage $(H=27.32, p<0.0001)$, and in the CERAD score $(H=25.50, p<0.0001)$ (Fig. 7). After post hoc analysis, there was a significant difference in CSF Ng between stages 

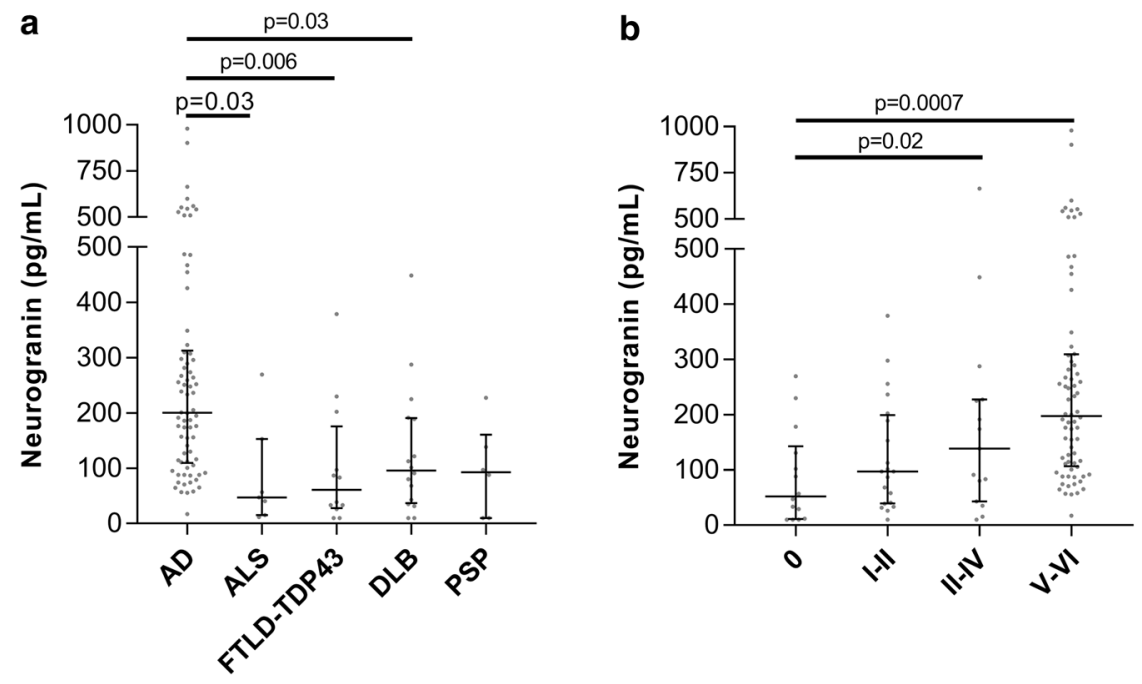

C
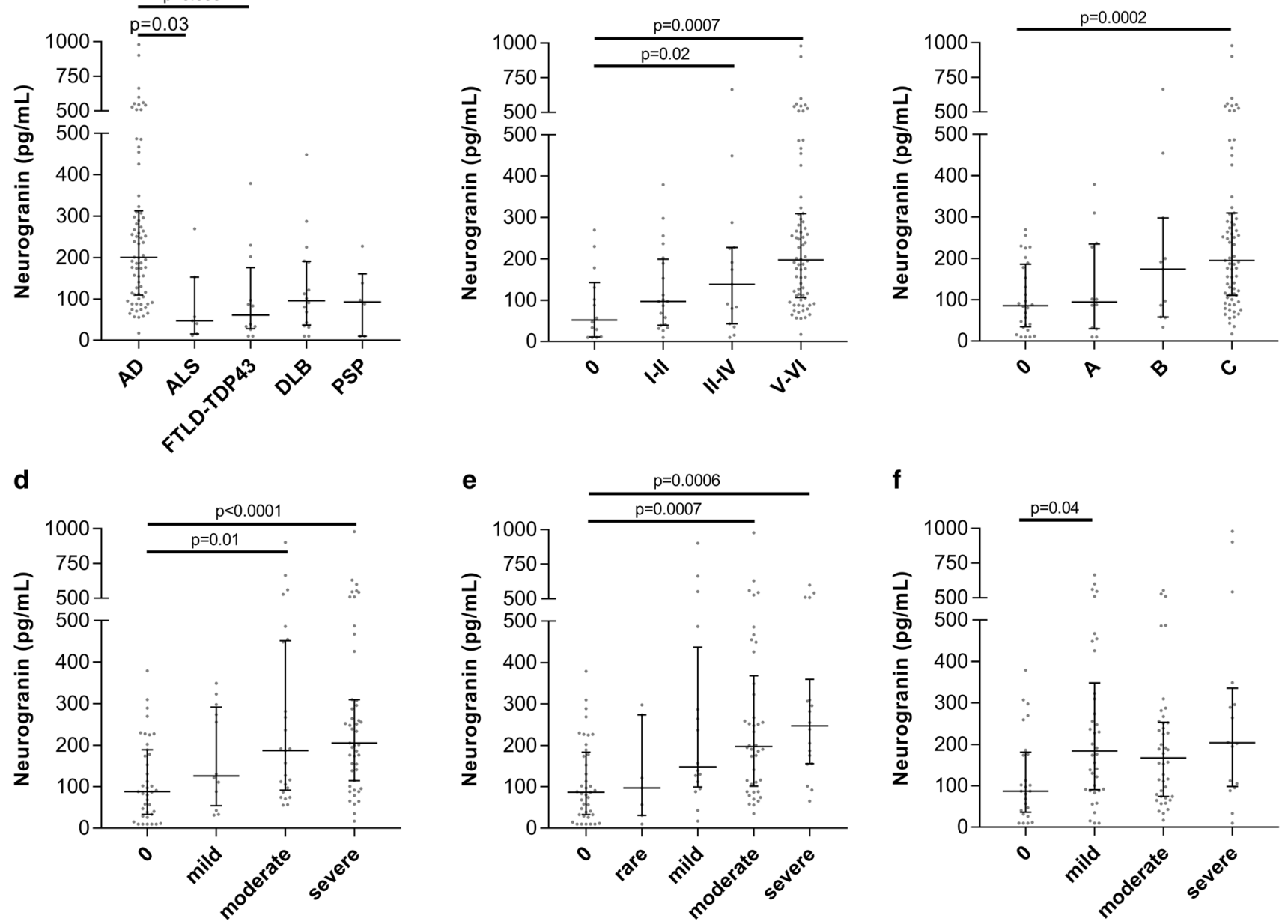

Fig. 6 Scatterplots displaying the CSF $\mathrm{Ng}$ concentrations in relation to a autopsy confirmed cases, $\mathbf{b}$ tau neurofibrillary tangles, $\mathbf{c} \mathrm{A} \beta$ neuritic plaques, $\mathbf{d} \mathrm{A} \beta$ neuritic plaques in amygdala, e $\mathrm{A} \beta$ neuritic plaques in hippocampus, and $\mathbf{f}$ neuronal loss in hippocampus. The

\begin{tabular}{|c|c|c|c|c|}
\hline \multirow{2}{*}{$\begin{array}{c}\text { Neurogranin v AD pathology } \\
\mathbf{A}^{\mathbf{b}}\end{array}$} & $\mathbf{C}^{\mathbf{c}}$ & $\mathbf{4}$ or $\mathbf{1}$ & $\mathbf{2}$ & $\mathbf{3}$ \\
\hline $\mathbf{0}$ & $\mathbf{0}$ & 69.55 & 47.97 & 227.29 \\
\hline $\mathbf{1}$ & $\mathbf{0}$ or $\mathbf{1}$ & 112.88 & 158.00 & NA \\
\hline & $\mathbf{2}$ or $\mathbf{3}$ & NA & 182.70 & NA \\
\hline \multirow{2}{*}{$\mathbf{3}$} & Any C & 156.75 & 93.46 & 127.39 \\
\hline \multirow{3}{*}{} & $\mathbf{0}$ or $\mathbf{1}$ & 236.77 & NA & 87.93 \\
\cline { 2 - 5 } & $\mathbf{2}$ or $\mathbf{3}$ & 96.92 & 314.83 & 257.55 \\
\hline
\end{tabular}

Fig. 7 (a) A heat map displaying the mean CSF Ng concentrations for 114 autopsy-confirmed subjects as defined by the ABC classification scheme. The ABC score incorporates histopathologic assessments of $A \beta$ deposits (A), staging of neurofibrillary tangles (B), and scoring of neuritic plaques (C). ${ }^{\text {a }}$ NFT stage should be determined by the method of Braak [5,6], ${ }^{\mathrm{b}} \mathrm{A} \beta /$ amyloid plaque score should be determined by the method of Thal et al. [49], ${ }^{\mathrm{c}}$ Neuritic plaque score should be determined by the method of CERAD [35] bars presented in the figures are medians with interquartile ranges and comparisons between groups were performed using Kruskal-Wallis test, followed by the Mann-Whitney $U$ test

For Braak staging, there was a significant difference in CSF $\mathrm{Ng}$ between stages 0 and $3(p<0.0001)$ and between stages 1 and $3(p=0.002)$, along with a significant difference between stages 0 and 2 which was no longer significant after multiple comparisons correction ( $p=0.04$ before, $p=0.18$ after). Finally, in the CERAD scheme there was a significant difference in CSF Ng between stages 0 and $3(p<0.0001)$ and a nearly significant difference between stages 1 and 3 $(p=0.054)$.

\section{Discussion}

Here, we report on CSF Ng concentrations in both clinically diagnosed and in neuropathologically confirmed subjects, and show that CSF Ng concentrations were significantly 
higher in ADD compared to DLB, FTD and ALS. Further, increased CSF Ng concentrations correlated with increased $\mathrm{A} \beta$ plaque load, specifically in the hippocampus and amygdala, probably reflecting synaptic damage induced by aggregation of $A \beta$ and accumulation in plaques.

CSF Ng is a well-replicated biomarker for ADD (http:// www.alzforum.org/alzbiomarker). As $\mathrm{Ng}$ is expressed in dendrites and as CSF Ng concentrations correlate with memory impairment and reduced cerebral glucose metabolism in ADD-affected brain regions [39], this biomarker has been proposed to reflect synaptic dysfunction in ADD. Importantly, CSF Ng appears to be an ADD-specific biomarker; its concentration is unaltered or even reduced in several neurodegenerative diseases, including FTD, PD and atypical parkinsonian disorders $[25,41,53]$. Here, we re-examine the ADD specificity of CSF Ng in a large cross-sectional serie of neurodegenerative diseases. We extended the analysis by examining the marker in pathologically confirmed cases and assessed its correlation with several pathological changes in the brain.

A common feature in several neurodegenerative diseases affecting memory is the presence of brain amyloidosis, including plaques (A $\beta$ ), tangles (tau) and Lewy bodies $(\alpha$-synuclein), and neuropathological studies have shown that ADD patients often have other concomitant pathologies, besides plaques and tangles [12, 24, 37]. We found increased CSF Ng concentrations with increased Thal, CERAD and Braak scores demonstrating that $\mathrm{Ng}$ is linked to the ADD pathology. Our results also suggest that CSF Ng values began to differentiate relatively earlier in Braak staging (between stages 0 and 2) compared to the others, while CSF Ng differentiation between stages persisted relatively late in Thal staging (between stages 2 and 3). CSF Ng concentrations differentiated least in CERAD scores compared to the others. We also show that increased plaque load in the hippocampus and amygdala was paralleled by increased CSF $\mathrm{Ng}$ concentrations while there was no association between tau, $\alpha$-synuclein or TDP-43 pathology and CSF Ng. These data support the hypothesis that $\mathrm{A} \beta$ neurotoxicity specifically affects synaptic function, and that plaque pathology associated with cognition can be translated into high CSF $\mathrm{Ng}$ concentrations. In addition, we found a weak negative correlation between CSF A $\beta$ and CSF Ng concentrations in the ADD group. These data further support the association between CSF Ng and plaque load. The deposition of A $\beta$ into plaques leads to a lowering of CSF A $\beta 42$ concentrations, while CSF Ng is believed to increase as a consequence of synaptic dysfunction and degeneration. There was also an association between neuronal loss in hippocampus and CSF $\mathrm{Ng}$ in the groups with negligible ADD neuropathology and low-level ADD which may reflect that the neuronal loss at early stages is most dynamic. However, this needs to be further evaluated and confirmed in additional studies.
We found that CSF Ng concentrations in ADD were higher in patients having one or two copies of the $A P O E$ $\varepsilon 4$ allele compared to non-carriers. The same trend was observed for the MCI group but there was no statistically significant difference between the groups, which may be due to low number of patients included. This is in agreement with a previous study and shows that the number of copies of the $\varepsilon 4$ allele may be associated with synaptic dysfunction which is reflected in the CSF as higher $\mathrm{Ng}$ concentrations [47]. One explanation could be that ADD patients with two copies of the $\varepsilon 4$ allele have an increased plaque load compared to patients with no $\varepsilon 4$ allele [11]. Thus, the number of $\varepsilon 4$ allele copies affects the amount of plaques (and/or neurotoxic $\mathrm{A} \beta$ species) which in turn induces synaptic pathology.

There was a significant association between the CSF Ng concentration and rate of cognitive decline, as measured by drop in MMSE scores per year in the MCI group. This is in agreement with previous studies [27] again showing that $\mathrm{Ng}$ is linked to synaptic function and cognition at early stages.

We show that increased CSF Ng concentration occurs only in ADD and not in the other neurodegenerative disorders investigated in this study. One exception was lvPPA which displayed similar CSF $\mathrm{Ng}$ concentrations as those found in ADD. A plausible explanation is that a large number of lvPPA patients actually have ADD pathology [20,33]. However, there were no correlations between CSF p-tau, t-tau, or A $\beta 42$ with CSF Ng in the lvPPA group. Future studies should investigate pathology-confirmed lvPPA cases with regional plaque distribution to establish if this finding still holds.

The CSF Ng concentrations were significantly increased in ADD compared to svPPA and nfvPNFA but also increased in lvPPA compared to bvFTD. This may be explanted by that svPPA and nfvPNFA are more predictive of FTLD-TDP and FTLD-tau, respectively. Thus, the finding reflects that lvPPA patients are likely atypical variants of ADD [13]. In addition, out of the 12 lvPPA patients included in the study, ten had available CSF A $\beta 42$ and t-tau concentrations measurements and eight of these were ADD biomarker positive, further supporting this statement.

bvFTD and PPA are diagnosed according the appearance of the symptoms. In the present study, CSF Ng concentrations in bvFTD were significantly lower than in ADD and lvPPA but also lower compared to CTRL which is in agreement with a previous study [53]. In addition, there were no correlations between CSF Ng and the core CSF ADD biomarkers in bvFTD. However, adding CSF Ng to the core AD biomarkers ( $\mathrm{A} \beta, \mathrm{t}$-tau and $\mathrm{p}$-tau) significantly increased the accuracy in distinguishing between CTRL and bvFTD and MCI vs bvFTD. Thus, adding CSF Ng to the core AD biomarkers (A $\beta$, t-tau and $\mathrm{p}$-tau) adds diagnostic information. Further studies are warranted to confirm these findings since 
the pathological heterogeneity of bvFTD may contribute to the results [22].

Interestingly, there was a strong positive correlation between CSF A $\beta 42$ and Ng levels in the ALS group and at the same time, CSF Ng concentrations were significantly lower in ALS compared to ADD and even slightly lower than in CTRL. It is known that a small percentage of ALS patients have ADD pathology [7] and it was shown recently that both amyloid precursor protein (APP) and intracellular $A \beta$ are overexpressed in the hippocampus in ALS compared to CTRL [14]. In animal models of ADD it has been shown that increasing the soluble APP fragment cleaved by $\alpha$-secretase may improve cognition and rescue long-term potentiation (LTP) $[34,38] . \mathrm{Ng}$ has also been shown to be associated with cognition and to play a role in LTP [18, 36, 54]. In addition, there was no correlation between neuritic plaque load and the CSF Ng concentrations in ALS. Thus, it is tempting to speculate that APP is involved in cell survival in ALS and that increased APP and $\mathrm{A} \beta$ expression in ALS, without plaque pathology, is reflected as low concentrations of CSF $\mathrm{Ng}$ which in turn reflect prosperous synapses. Further studies are warranted to confirm these findings.

When evaluating novel CSF biomarkers for ADD, it is important to consider that concomitant pathologies are common, especially in elderly patients with dementia. To test CSF Ng as a specific biomarker for ADD, we investigated its performance in ADD and other neurodegenerative disease cases with definitive diagnoses determined by postmortem examination. However, the number of patients was low in some groups. Thus, larger studies including neuropathology confirmed patients are warranted.

The study has a number of limitations that should be acknowledged. First, the sample size in some subgroups was small and the risk of false-positive statistical test warrants replication of the most interesting findings. Therefore, interpretation should be made with caution. Second, although our study replicates the specificity of CSF Ng for $\mathrm{ADD}$, a number of novel findings regarding what CSF $\mathrm{Ng}$ may add to other markers need to be replicated in independent cohorts. Thus, in the future, we will seek verification of these results in other, independent datasets to investigate their clinical relevance.

Some of the highest CSF Ng levels were found in two individuals in the CTRL group. Data from neuropathological examination were not available and, therefore, it is unknown whether these high levels are due to that these individuals have $\mathrm{AD}$, or possibly other forms of pathology, a change in the overall clearance and production of $\mathrm{Ng}$, or possibly if the very high values have analytical or technical explanations.

In conclusion, CSF Ng is a biomarker specifically reflecting synaptic pathology in ADD and its concentration is linked to the extent of plaque pathology in the hippocampus and amygdala. The findings support the use of CSF Ng as a biomarker for diagnosing ADD and also for treatment trials, where disease-modifying drugs are evaluated, to monitor if treatment restores synaptic function in the patients.

Acknowledgements The study was supported by grants from the Swedish and European Research Councils, the Torsten Söderberg Foundation, the Swedish Brain Foundation, the Knut and Alice Wallenberg Foundation, Frimurarestiftelsen, Stiftelsen för Gamla Tjänarinnor, Foundation for Research on Alzheimer, the Swedish Alzheimer Foundation, Swedish State Support for Clinical Research (ALFGBG) in addition to the National Institutes of Health (P30 AG-10124-27, P01 AG-17586-18, P50 NS-053488-11, and NS088341).

\section{Compliance with ethical standards}

Conflict of interest EP, LMS, NCC, ACP, DW, ÅS, UA, KH, HK, DJI, LE, LM, BO, JBT, and MG declare that they have no conflict of interest. DAW received grant funding from Merck, Biogen, Avid Radiopharmaceuticals and Eli Lilly and personal fees from GE Healthcare, Merck, and Janssen. HZ is a co-founder of Brain Biomarker Solutions in Gothenburg AB, a GU Venture-based platform company at the University of Gothenburg, has served at advisory boards of Eli Lilly and Roche Diagnostics and has received travel support from TEVA. KB has served as a consultant or at advisory boards for Alzheon, BioArctic, Biogen, Eli Lilly, Fujirebio Europe, IBL International, Merck, Pfizer, and Roche Diagnostics, and is a co-founder of Brain Biomarker Solutions in Gothenburg AB, a GU Venture-based platform company at the University of Gothenburg.

Open Access This article is distributed under the terms of the Creative Commons Attribution 4.0 International License (http://creativeco mmons.org/licenses/by/4.0/), which permits unrestricted use, distribution, and reproduction in any medium, provided you give appropriate credit to the original author(s) and the source, provide a link to the Creative Commons license, and indicate if changes were made.

\section{References}

1. Armstrong MJ, Litvan I, Lang AE, Bak TH, Bhatia KP, Borroni $B$ et al (2013) Criteria for the diagnosis of corticobasal degeneration. Neurology 80:496-503. https://doi.org/10.1212/WNL.0b013 e31827f0fd 1

2. Bertoni-Freddari C, Fattoretti P, Casoli T, Caselli U, Meier-Ruge W (1996) Deterioration threshold of synaptic morphology in aging and senile dementia of Alzheimer's type. Anal Quant Cytol Histol 18:209-213

3. Blennow K, Bogdanovic N, Alafuzoff I, Ekman R, Davidsson P (1996) Synaptic pathology in Alzheimer's disease: relation to severity of dementia, but not to senile plaques, neurofibrillary tangles, or the ApoE4 allele. J Neural Transm (Vienna) 103:603-618. https://doi.org/10.1007/BF01273157

4. Bogdanovic N, Davidsson P, Gottfries J, Volkman I, Winblad B, Blennow K (2002) Regional and cellular distribution of synaptic proteins in the normal human brain. Brain Aging 2:18-30

5. Braak H, Braak E (1991) Neuropathological stageing of Alzheimer-related changes. Acta Neuropathol 82:239-259

6. Braak H, Alafuzoff I, Arzberger T, Kretzschmar H, Del Tredici K (2006) Staging of Alzheimer disease-associated neurofibrillary pathology using paraffin sections and immunocytochemistry. 
Acta Neuropathol 112:389-404. https://doi.org/10.1007/s0040 1-006-0127-z

7. Brettschneider J, Libon DJ, Toledo JB, Xie SX, McCluskey L, Elman L et al (2012) Microglial activation and TDP-43 pathology correlate with executive dysfunction in amyotrophic lateral sclerosis. Acta Neuropathol 123:395-407. https://doi.org/10.1007/ s00401-011-0932-X

8. Davidsson P, Puchades M, Blennow K (1999) Identification of synaptic vesicle, pre- and postsynaptic proteins in human cerebrospinal fluid using liquid-phase isoelectric focusing. Electrophoresis 20:431-437. https://doi.org/10.1002/ (sici)1522-2683(19990301)20:3<431::aid-elps431>3.0.co;2-2

9. Davies CA, Mann DM, Sumpter PQ, Yates PO (1987) A quantitative morphometric analysis of the neuronal and synaptic content of the frontal and temporal cortex in patients with Alzheimer's disease. J Neurol Sci 78:151-164

10. DeKosky ST, Scheff SW (1990) Synapse loss in frontal cortex biopsies in Alzheimer's disease: correlation with cognitive severity. Ann Neurol 27:457-464. https://doi.org/10.1002/ana.41027 0502

11. Drzezga A, Grimmer T, Henriksen G, Muhlau M, Perneczky R, Miederer I et al (2009) Effect of APOE genotype on amyloid plaque load and gray matter volume in Alzheimer disease. Neurology 72:1487-1494. https://doi.org/10.1212/WNL.0b013e3181 a2e8d0

12. Franklin EE, Perrin RJ, Vincent B, Baxter M, Morris JC, Cairns $\mathrm{NJ}$ et al (2015) Brain collection, standardized neuropathologic assessment, and comorbidity in Alzheimer's disease neuroimaging initiative 2 participants. Alzheimers Dement 11:815-822. https:// doi.org/10.1016/j.jalz.2015.05.010

13. Giannini LAA, Irwin DJ, McMillan CT, Ash S, Rascovsky K, Wolk DA et al (2017) Clinical marker for Alzheimer disease pathology in logopenic primary progressive aphasia. Neurology 88:2276-2284. https://doi.org/10.1212/WNL.000000000000403 4

14. Gomez-Pinedo U, Villar-Quiles RN, Galan L, Matias-Guiu JA, Benito-Martin MS, Guerrero-Sola A et al (2016) Immununochemical markers of the amyloid cascade in the hippocampus in motor neuron diseases. Front Neurol 7:195. https://doi.org/10.3389/fneur .2016 .00195

15. Gorno-Tempini ML, Hillis AE, Weintraub S, Kertesz A, Mendez M, Cappa SF et al (2011) Classification of primary progressive aphasia and its variants. Neurology 76:1006-1014. https://doi. org/10.1212/WNL.0b013e31821103e6

16. Guadano-Ferraz A, Vinuela A, Oeding G, Bernal J, Rausell E (2005) RC3/neurogranin is expressed in pyramidal neurons of motor and somatosensory cortex in normal and denervated monkeys. J Comp Neurol 493:554-570. https://doi.org/10.1002/ cne. 20774

17. Hoglinger GU, Respondek G, Stamelou M, Kurz C, Josephs KA, Lang AE et al (2017) Clinical diagnosis of progressive supranuclear palsy: the movement disorder society criteria. Mov Disord 32:853-864. https://doi.org/10.1002/mds.26987

18. Huang KP, Huang FL, Jager T, Li J, Reymann KG, Balschun D (2004) Neurogranin/RC3 enhances long-term potentiation and learning by promoting calcium-mediated signaling. J Neurosci 24:10660-10669. https://doi.org/10.1523/JNEUR OSCI.2213-04.2004

19. Hyman BT, Phelps CH, Beach TG, Bigio EH, Cairns NJ, Carrillo MC et al (2012) National Institute on Aging-Alzheimer's Association guidelines for the neuropathologic assessment of Alzheimer's disease. Alzheimers Dement 8:1-13. https://doi.org/10.1016/j. jalz.2011.10.007

20. Irwin DJ, Cairns NJ, Grossman M, McMillan CT, Lee EB, Van Deerlin VM et al (2015) Frontotemporal lobar degeneration: defining phenotypic diversity through personalized medicine. Acta Neuropathol 129:469-491. https://doi.org/10.1007/s0040 1-014-1380-1

21. Irwin DJ, Grossman M, Weintraub D, Hurtig HI, Duda JE, Xie SX et al (2017) Neuropathological and genetic correlates of survival and dementia onset in synucleinopathies: a retrospective analysis. Lancet Neurol 16:55-65. https://doi.org/10.1016/S1474 $-4422(16) 30291-5$

22. Irwin DJ, Lleo A, Xie SX, McMillan CT, Wolk DA, Lee EB et al (2017) Ante mortem cerebrospinal fluid tau levels correlate with postmortem tau pathology in frontotemporal lobar degeneration. Ann Neurol 82:247-258. https://doi.org/10.1002/ana.24996

23. Irwin DJ, White MT, Toledo JB, Xie SX, Robinson JL, Van Deerlin V et al (2012) Neuropathologic substrates of Parkinson disease dementia. Ann Neurol 72:587-598. https://doi.org/10.1002/ ana. 23659

24. James BD, Wilson RS, Boyle PA, Trojanowski JQ, Bennett DA, Schneider JA (2016) TDP-43 stage, mixed pathologies, and clinical Alzheimer's-type dementia. Brain. https://doi.org/10.1093/ brain/aww224

25. Janelidze S, Hertze J, Zetterberg H, Landqvist Waldo M, Santillo A, Blennow $\mathrm{K}$ et al (2016) Cerebrospinal fluid neurogranin and YKL-40 as biomarkers of Alzheimer's disease. Ann Clin Transl Neurol 3:12-20. https://doi.org/10.1002/acn3.266

26. Kester MI, Teunissen CE, Crimmins DL, Herries EM, Ladenson JH, Scheltens P et al (2015) Neurogranin as a cerebrospinal fluid biomarker for synaptic loss in symptomatic Alzheimer disease. JAMA Neurol 72:1275-1280. https://doi.org/10.1001/jamaneurol .2015 .1867

27. Kvartsberg H, Duits FH, Ingelsson M, Andreasen N, Ohrfelt A, Andersson $\mathrm{K}$ et al (2015) Cerebrospinal fluid levels of the synaptic protein neurogranin correlates with cognitive decline in prodromal Alzheimer's disease. Alzheimers Dement 11:1180-1190. https:// doi.org/10.1016/j.jalz.2014.10.009

28. Kvartsberg H, Portelius E, Andreasson U, Brinkmalm G, Hellwig $\mathrm{K}$, Lelental $\mathrm{N}$ et al (2015) Characterization of the postsynaptic protein neurogranin in paired cerebrospinal fluid and plasma samples from Alzheimer's disease patients and healthy controls. Alzheimers Res Ther 7:40. https://doi.org/10.1186/s1319 5-015-0124-3

29. Masliah E, Mallory M, Alford M, DeTeresa R, Hansen LA, McKeel DW Jr et al (2001) Altered expression of synaptic proteins occurs early during progression of Alzheimer's disease. Neurology $56: 127-129$

30. McKeith IG, Dickson DW, Lowe J, Emre M, O'Brien JT, Feldman $\mathrm{H}$ et al (2005) Diagnosis and management of dementia with Lewy bodies: third report of the DLB Consortium. Neurology 65:1863-1872. https://doi.org/10.1212/01.wnl.0000187889.17253 .$b 1$

31. McKhann G, Drachman D, Folstein M, Katzman R, Price D, Stadlan EM (1984) Clinical diagnosis of Alzheimer's disease: report of the NINCDS-ADRDA Work Group under the auspices of Department of Health and Human Services Task Force on Alzheimer's Disease. Neurology 34:939-944

32. McKhann GM, Knopman DS, Chertkow H, Hyman BT, Jack CR Jr, Kawas CH et al (2011) The diagnosis of dementia due to Alzheimer's disease: recommendations from the National Institute on Aging-Alzheimer's Association workgroups on diagnostic guidelines for Alzheimer's disease. Alzheimers Dement 7:263-269. https://doi.org/10.1016/j.jalz.2011.03.005

33. Mesulam MM, Rogalski EJ, Wieneke C, Hurley RS, Geula C, Bigio EH et al (2014) Primary progressive aphasia and the evolving neurology of the language network. Nat Rev Neurol 10:554569. https://doi.org/10.1038/nrneurol.2014.159 
34. Meziane H, Dodart JC, Mathis C, Little S, Clemens J, Paul SM et al (1998) Memory-enhancing effects of secreted forms of the beta-amyloid precursor protein in normal and amnestic mice. Proc Natl Acad Sci USA 95:12683-12688

35. Mirra SS, Heyman A, McKeel D, Sumi SM, Crain BJ, Brownlee LM et al. (1991) The Consortium to Establish a Registry for Alzheimer's Disease (CERAD). Part II. Standardization of the neuropathologic assessment of Alzheimer's disease. Neurology 41:479-486

36. Mons N, Enderlin V, Jaffard R, Higueret P (2001) Selective agerelated changes in the $\mathrm{PKC}$-sensitive, calmodulin-binding protein, neurogranin, in the mouse brain. J Neurochem 79:859-867

37. Montine TJ, Phelps CH, Beach TG, Bigio EH, Cairns NJ, Dickson DW et al (2012) National Institute on Aging-Alzheimer's Association guidelines for the neuropathologic assessment of Alzheimer's disease: a practical approach. Acta Neuropathol 123:1-11. https ://doi.org/10.1007/s00401-011-0910-3

38. Moreno L, Rose C, Mohanraj A, Allinquant B, Billard JM, Dutar P (2015) sAbetaPPalpha improves hippocampal NMDA-dependent functional alterations linked to healthy aging. J Alzheimers Dis 48:927-935. https://doi.org/10.3233/JAD-150297

39. Portelius E, Zetterberg H, Skillback T, Tornqvist U, Andreasson U, Trojanowski JQ et al (2015) Cerebrospinal fluid neurogranin: relation to cognition and neurodegeneration in Alzheimer's disease. Brain 138:3373-3385. https://doi.org/10.1093/brain/awv26 7

40. Rascovsky K, Hodges JR, Knopman D, Mendez MF, Kramer JH, Neuhaus J et al (2011) Sensitivity of revised diagnostic criteria for the behavioural variant of frontotemporal dementia. Brain 134:2456-2477. https://doi.org/10.1093/brain/awr179

41. Remnestal J, Just D, Mitsios N, Fredolini C, Mulder J, Schwenk JM et al (2016) CSF profiling of the human brain enriched proteome reveals associations of neuromodulin and neurogranin to Alzheimer's disease. Proteom Clin Appl 10:1242-1253. https:// doi.org/10.1002/prca.201500150

42. Represa A, Deloulme JC, Sensenbrenner M, Ben-Ari Y, Baudier J (1990) Neurogranin: immunocytochemical localization of a brainspecific protein kinase C substrate. J Neurosci 10:3782-3792

43. Sanfilippo C, Forlenza O, Zetterberg H, Blennow K (2016) Increased neurogranin concentrations in cerebrospinal fluid of Alzheimer's disease and in mild cognitive impairment due to AD. J Neural Transm (Vienna) 123:1443-1447. https://doi. org/10.1007/s00702-016-1597-3

44. Shahim P, Tegner Y, Gustafsson B, Gren M, Arlig J, Olsson M et al (2016) Neurochemical aftermath of repetitive mild traumatic brain injury. JAMA Neurol 73:1308-1315. https://doi. org/10.1001/jamaneurol.2016.2038

45. Shaw LM, Vanderstichele H, Knapik-Czajka M, Clark CM, Aisen PS, Petersen RC et al (2009) Cerebrospinal fluid biomarker signature in Alzheimer's disease neuroimaging initiative subjects. Ann Neurol 65:403-413. https://doi.org/10.1002/ana.21610

46. Strong MJ, Abrahams S, Goldstein LH, Woolley S, McLaughlin P, Snowden J et al (2017) Amyotrophic lateral sclerosis-frontotemporal spectrum disorder (ALS-FTSD): revised diagnostic criteria. Amyotroph Lateral Scler Frontotemporal Degener 18:153-174. https://doi.org/10.1080/21678421.2016.1267768

47. Sun X, Dong C, Levin B, Crocco E, Loewenstein D, Zetterberg H et al (2016) APOE epsilon4 carriers may undergo synaptic damage conferring risk of Alzheimer's disease. Alzheimers Dement 12:1159-1166. https://doi.org/10.1016/j.jalz.2016.05.003

48. Sze CI, Troncoso JC, Kawas C, Mouton P, Price DL, Martin LJ (1997) Loss of the presynaptic vesicle protein synaptophysin in hippocampus correlates with cognitive decline in Alzheimer disease. J Neuropathol Exp Neurol 56:933-944

49. Thal DR, Rub U, Orantes M, Braak H (2002) Phases of A betadeposition in the human brain and its relevance for the development of AD. Neurology 58:1791-1800

50. Thorsell A, Bjerke M, Gobom J, Brunhage E, Vanmechelen E, Andreasen N et al (2010) Neurogranin in cerebrospinal fluid as a marker of synaptic degeneration in Alzheimer's disease. Brain Res 1362:13-22. https://doi.org/10.1016/j.brainres.2010.09.073

51. Toledo JB, Brettschneider J, Grossman M, Arnold SE, Hu WT, Xie SX et al (2012) CSF biomarkers cutoffs: the importance of coincident neuropathological diseases. Acta Neuropathol 124:2335. https://doi.org/10.1007/s00401-012-0983-7

52. Toledo JB, Van Deerlin VM, Lee EB, Suh E, Baek Y, Robinson JL et al (2014) A platform for discovery: the University of Pennsylvania Integrated Neurodegenerative Disease Biobank. Alzheimers Dement 10(477-484):e471. https://doi.org/10.1016/j. jalz.2013.06.003

53. Wellington H, Paterson RW, Portelius E, Tornqvist U, Magdalinou $\mathrm{N}$, Fox NC et al (2016) Increased CSF neurogranin concentration is specific to Alzheimer disease. Neurology 86:829-835. https:// doi.org/10.1212/WNL.0000000000002423

54. Wu J, Li J, Huang KP, Huang FL (2002) Attenuation of protein kinase $\mathrm{C}$ and cAMP-dependent protein kinase signal transduction in the neurogranin knockout mouse. J Biol Chem 277:1949819505. https://doi.org/10.1074/jbc.M109082200

55. Xie SX, Baek Y, Grossman M, Arnold SE, Karlawish J, Siderowf A et al (2011) Building an integrated neurodegenerative disease database at an academic health center. Alzheimers Dement 7:e84e93. https://doi.org/10.1016/j.jalz.2010.08.233

56. Yoshiyama Y, Higuchi M, Zhang B, Huang SM, Iwata N, Saido TC et al (2007) Synapse loss and microglial activation precede tangles in a P301S tauopathy mouse model. Neuron 53:337-351. https://doi.org/10.1016/j.neuron.2007.01.010

\section{Affiliations}

\section{Erik Portelius $^{1,2}$ - Bob Olsson ${ }^{1,2} \cdot$ Kina Höglund $^{1,2} \cdot$ Nicholas C. Cullen $^{1} \cdot$ Hlin Kvartsberg $^{1} \cdot$ Ulf Andreasson $^{1,2}$. Henrik Zetterberg ${ }^{1,2,3,4}$. Åsa Sandelius ${ }^{1}$. Leslie M. Shaw ${ }^{5}$. Virginia M. Y. Lee ${ }^{5}$. David J. Irwin ${ }^{6} \cdot$ Murray Grossman $^{6}$. Daniel Weintraub ${ }^{7,8}$. Alice Chen-Plotkin ${ }^{6}$. David A. Wolk ${ }^{6}$. Leo McCluskey ${ }^{6}$. Lauren Elman ${ }^{6}$. Jennifer McBride ${ }^{5}$. Jon B. Toledo ${ }^{5,9} \cdot$ John Q. Trojanowski ${ }^{5} \cdot$ Kaj Blennow $^{1,2}$}

1 Department of Psychiatry and Neurochemistry, Institute of Neuroscience and Physiology, The Sahlgrenska Academy at the University of Gothenburg, 431 80, Mölndal, Sweden

2 Clinical Neurochemistry Laboratory, Sahlgrenska University Hospital, Mölndal, Sweden
3 Department of Molecular Neuroscience, UCL Institute of Neurology, Queen Square, London WC1E 6BT, UK

4 UK Dementia Research Institute, London WC1E 6BT, UK

5 Department of Pathology and Laboratory Medicine, Institute on Aging, Center for Neurodegenerative Disease Research, 
University of Pennsylvania School of Medicine, Philadelphia, PA, USA

6 Department of Neurology, University of Pennsylvania School of Medicine, Philadelphia, PA, USA

7 Department of Psychiatry, University of Pennsylvania School of Medicine, Philadelphia, PA, USA
8 Parkinson's Disease and Mental Illness Research, Education and Clinical Centers (PADRECC and MIRECC), Philadelphia Veterans Affairs Medical Center, Philadelphia, PA, USA

9 Department of Neurology, Houston Methodist Hospital, Houston, TX, USA 